\title{
Child-onset thrombotic thrombocytopenic purpura caused by p.R498C and p.G259PfsX133 mutations in ADAMTS13
}

Citation for published version (APA):

Schelpe, A-S., Orlando, C., Ercig, B., Geeroms, C., Pareyn, I., Vandeputte, N., Pereira, L. C., Roose, E., Fostier, K., Nicolaes, G. A. F., Deckmyn, H., De Meyer, S. F., Vanhoorelbeke, K., \& Jochmans, K. (2018). Child-onset thrombotic thrombocytopenic purpura caused by p.R498C and p.G259PfsX133 mutations in ADAMTS13. European Journal of Haematology, 101(2), 191-199. https://doi.org/10.1111/ejh.13094

Document status and date:

Published: 01/08/2018

DOI:

10.1111/ejh.13094

Document Version:

Publisher's PDF, also known as Version of record

Document license:

Taverne

Please check the document version of this publication:

- A submitted manuscript is the version of the article upon submission and before peer-review. There can be important differences between the submitted version and the official published version of record.

People interested in the research are advised to contact the author for the final version of the publication, or visit the DOI to the publisher's website.

- The final author version and the galley proof are versions of the publication after peer review.

- The final published version features the final layout of the paper including the volume, issue and page numbers.

Link to publication

\footnotetext{
General rights rights.

- You may freely distribute the URL identifying the publication in the public portal. please follow below link for the End User Agreement:

www.umlib.nl/taverne-license

Take down policy

If you believe that this document breaches copyright please contact us at:

repository@maastrichtuniversity.nl

providing details and we will investigate your claim.
}

Copyright and moral rights for the publications made accessible in the public portal are retained by the authors and/or other copyright owners and it is a condition of accessing publications that users recognise and abide by the legal requirements associated with these

- Users may download and print one copy of any publication from the public portal for the purpose of private study or research.

- You may not further distribute the material or use it for any profit-making activity or commercial gain

If the publication is distributed under the terms of Article $25 \mathrm{fa}$ of the Dutch Copyright Act, indicated by the "Taverne" license above, 


\title{
Child-onset thrombotic thrombocytopenic purpura caused by p.R498C and p.G259PfsX133 mutations in ADAMTS13
}

\author{
An-Sofie Schelpe $^{1}$ (1) | Christelle Orlando ${ }^{2}$ | Bogac Ercig ${ }^{3,4,5}$ | Chloë Geeroms ${ }^{1}$ | \\ Inge Pareyn $^{1}$ | Nele Vandeputte ${ }^{1}$ | Leydi Carolina Velásquez Pereira ${ }^{1}$ | Elien Roose ${ }^{1}$ | \\ Karel Fostier $^{2}$ | Gerry A.F. Nicolaes ${ }^{3,4}$ | Hans Deckmyn ${ }^{1}$ | Simon F. De Meyer ${ }^{1}$ | \\ Karen Vanhoorelbeke $^{1}$ | Kristin Jochmans ${ }^{2}$
}

${ }^{1}$ Laboratory for Thrombosis Research, IRF Life Sciences, KU Leuven Campus Kulak Kortrijk, Kortrijk, Belgium

${ }^{2}$ Department of Haematology, Universitair Ziekenhuis Brussel (UZ Brussel), Brussels, Belgium

${ }^{3}$ Department of Biochemistry,

Cardiovascular Research Institute Maastricht (CARIM), Maastricht University, Maastricht, The Netherlands

${ }^{4}$ PharmaTarget B.V., Maastricht, The Netherlands

${ }^{5}$ Department of Plasma Proteins, SanquinAMC Landsteiner Laboratory, Amsterdam, The Netherlands

\section{Correspondence}

Karen Vanhoorelbeke, Laboratory for Thrombosis Research, IRF Life Sciences, KU Leuven Campus Kulak Kortrijk, Kortrijk, Belgium.

Email: Karen.Vanhoorelbeke@kuleuven.be

Funding information

This manuscript was supported by the following grant(s): the KU Leuven grants OT/14/071 and PF/10/014, and the European Framework Program for Research and Innovation (Horizon2020 Marie Sklodowska Curie Innovative training network PROFILE grant 675746). AnSofie Schelpe is supported by a PhD grant from the Agency Innovation And Entrepreneurship (VLAIO, www.iwt.be), Flanders, Belgium (141136).

\begin{abstract}
Introduction: Patients suffering from congenital thrombotic thrombocytopenic purpura (cTTP) have a deficiency in ADAMTS13 due to mutations in their ADAMTS13 gene.

Objective: The aim of this study was to determine ADAMTS13 parameters (activity, antigen, and mutations), to investigate if the propositus suffered from child-onset cTTP, and to study the in vitro effect of the ADAMTS13 mutations.

Methods: ADAMTS13 activity and antigen were determined using the FRETS VWF73 assay and ELISA and ADAMTS13 mutations via sequencing of the exons. Mutant proteins were expressed in Chinese hamster ovary cells, and their expression was studied using fluorescence microscopy and ELISA. Molecular modeling was used to evaluate the effect of the mutations on ADAMTS13 structure and stability.

Results: The propositus was diagnosed with cTTP at the age of 20. ADAMTS13 activity was below $10 \%$, and 2 compound heterozygous mutations, the p.R498C point and the p.G259PfsX133 frameshift mutation, were identified. Expression of ADAMTS13 mutants revealed that the p.R498C and the p.G259PfsX133 mutation cause secretion and translation defects in vitro, respectively. Molecular modeling showed that the R498 intra-domain interactions are lacking in the p.R498C mutant, resulting in protein instability.

Conclusion: The ADAMTS13 mutations result in a severe ADAMTS13 deficiency explaining the patient's phenotype.

KEYWORDS

ADAMTS13, mutation, thrombotic thrombocytopenic purpura
\end{abstract}

\section{1 | INTRODUCTION}

Patients suffering from thrombotic thrombocytopenic purpura (TTP) present with severe thrombocytopenia and microangiopathic hemolytic anemia, often associated with organ damage leading to neurological and cardiac symptoms and variable degrees of renal dysfunction. ${ }^{1,2}$ TTP is caused by a deficiency in ADAMTS13 (A Disintegrin And Metalloprotease with ThromboSpondin type 1 repeats, member 13), which leads to the accumulation of ultra-large (UL) von Willebrand factor (VWF) multimers in the circulation. The hyperactive UL-VWF multimers unfold and spontaneously bind platelets under shear, resulting in wide-spread platelet-rich micro 
thrombi that cause severe organ failure. ${ }^{1}$ ADAMTS13 deficiency can be caused by either mutations in the ADAMTS13 gene (congenital TTP, cTTP or Upshaw-Schulman syndrome) or the presence of antiADAMTS13 autoantibodies, inhibiting the enzymatic activity or accelerating ADAMTS13 clearance (acquired immune-mediated TTP, iTTP). Additional triggers such as infections, pregnancy, stress, and surgery often initiate an acute TTP event. Without prompt treatment, TTP is often fatal. ${ }^{1,2}$ ADAMTS13 replacement is the first-line therapy for TTP patients. Congenital TTP patients are treated with plasma infusion to restore functional ADAMTS13 levels, whereas ITTP patients are treated with plasma exchange to remove pathological anti-ADAMTS13 autoantibodies and to supply functional ADAMTS13. ${ }^{3}$

TTP is a rare and heterogeneous condition. Time of disease onset varies; $9 \%$ of patients experience their first acute TTP episode during childhood, whereas $91 \%$ of patients only during adulthood. ${ }^{2}$ The estimated annual prevalence of TTP is 10 cases per million, of which the majority is iTTP (95\%). The remaining $5 \%$ suffers of cTTP due to mutations in the ADAMTS13 gene, which are inherited in an autosomal recessive manner. Only patients carrying homozygous or compound heterozygous mutations are symptomatic. ${ }^{4}$ Since the discovery in 2001 that ADAMTS13 mutations are causative for TTP ${ }^{5}$, about 150 disease-causing mutations have been reported. ${ }^{2,6-10}$ Furthermore, polymorphisms might positively or negatively affect ADAMTS13 expression and in combination with ADAMTS13 mutations might additionally contribute to ADAMTS13 deficiency. ${ }^{11}$ Using in vitro expression studies, some ADAMTS13 mutations were shown to cause defects in protein secretion ${ }^{12-14}$ and/or a reduction in its activity. ${ }^{15,16}$

We here report the disease history of a male patient suffering from "infection-induced hemolytic anemia and thrombocytopenia" since the age of 1 with eventually, 19 years later, diagnosis of child-onset CTTP. We identified the disease-causing mutations; the p.G259PfsX133 frameshift and the p.R498C missense mutation, and their impact on the patient's phenotype.

\section{2 | MATERIALS AND METHODS}

\section{1 | Case history}

The now 30-year-old patient is the first child of non-consanguineous parents from Caucasian origin. He was born at 39.5 weeks of gestation after an uneventful pregnancy. At the age of 13 months, he was admitted to the hospital with fever and diarrhea. His skin had a subicteric aspect, and ecchymoses were present on his face, limbs, and chest. Laboratory analyses revealed severe thrombocytopenia (34 $\times 10^{9} / \mathrm{L}$, Table 1$)$, elevated lactate dehydrogenase (LDH, $1768 \mathrm{U} / \mathrm{mL}$, Table 1), and elevated bilirubin levels. Renal function was normal, and there were no signs of neurological impairment. Stool culture was positive for Yersinia enterocolitica and Escherichia coli, and colimycin treatment was started. In the following days, hemoglobin levels progressively dropped and packed red blood cells were transfused once. After 10 days of treatment, hematological parameters normalized and the patient was discharged with the diagnosis of "infection-induced hemolytic anemia and thrombocytopenia."

During the following 14 years, he experienced 5 similar episodes of thrombocytopenia and hemolytic anemia, all triggered by bacterial or viral infections (Table 1). Schistocytes were detected in the peripheral blood smears on multiple occasions. Pediatricians suggested the diagnosis of "chronic relapsing schistocytic hemolytic anemia and thrombocytopenia of infancy and childhood". Treatment consisted of administration of fresh frozen plasma (FFP), intravenous immunoglobulins, and/or platelet transfusion, according to the physician's judgment. Renal function remained mainly normal, and no thrombotic episodes occurred.

\section{2 | Samples}

Blood and plasma samples were collected at time of congenital TTP suspicion from the patient (episode 7, Table 1) and both parents for genetic analysis, ADAMTS13 characterization and anti-ADAMTS13 inhibitor testing. Plasma samples of the patient were available from 2 additional episodes (episode 8 and 10, Table 1) and during prophylactic FFP infusion ( every 3 weeks, samples from sessions 1 , 4, and 10; Table 1) for ADAMTS13 and VWF testing. Clinical parameters (if available) were given by UZ Brussel. Analyses were approved by the Ethical Committee of the KU Leuven and UZ Brussel. Informed consent was obtained in accordance with the Declaration of Helsinki.

\section{3 | Recombinant human ADAMTS13 and polyclonal rabbit anti-human ADAMTS13 antibodies}

Recombinant human (rh)ADAMTS13 with a C-terminal His- and V5-tag was produced as explained elsewhere. ${ }^{17}$ Purity of rhADAMTS13 was assessed by reducing sodium dodecyl sulfate polyacrylamide gel electrophoresis (SDS-PAGE) and Coomassie blue staining. Polyclonal rabbit anti-rhADAMTS13 antibodies were produced by immunizing a New Zealand rabbit with rhADAMTS13 as described. ${ }^{18}$ Twenty microgram of rhADAMTS13 in $100 \mu \mathrm{L}$ of complete Freund's adjuvant (Sigma, Missouri) was used for the first immunization of the rabbit (male, $1.5 \mathrm{~kg}$ ). After 2, 4, and 6 weeks, the immune system of the rabbit was boosted with $20 \mu \mathrm{g}$ of rhADAMTS13 in $100 \mu \mathrm{L}$ incomplete Freund's adjuvant (Sigma). Immunoglobulin G's (IgG) from rabbit serum were purified using Protein G Sepharose 4 Fast Flow (GE Healthcare) affinity chromatography, and dialyzed against phosphate-buffered saline (PBS). The polyclonal rabbit anti-rhADAMTS13 antibody was able to recognize the M, MD, MDT, MDTCS, and T2-C2 variant in ELISA (data not shown).

\section{4 | ADAMTS13 antigen ELISA}

ADAMTS13 antigen levels in plasma or expression medium were measured using an immunoassay as described ${ }^{19}$, with minor 
modifications. Briefly, a 96-well microtiter plate was coated with the in-house developed mouse anti-human ADAMTS13 antibody $3 \mathrm{H} 9^{20}$ (targeting the metalloprotease domain; $5 \mu \mathrm{g} / \mathrm{mL}$ ) in carbonate/bicarbonate coating buffer $\left(50 \mathrm{mmol} / \mathrm{L} \mathrm{Na}_{2} \mathrm{CO}_{3} / \mathrm{NaHCO}_{3}, \mathrm{pH}\right.$ 9.6). A 1.5 over 2.5 dilution series of plasma (start dilution $8 \%, \mathrm{v} / \mathrm{v}$ ) or expression medium containing rhADAMTS13 (start dilution $90 \%$, v/v) was added. Bound ADAMTS13 was detected by adding the inhouse developed polyclonal rabbit anti-rhADAMTS13 IgG's $(1.5 \mu \mathrm{g} /$ $\mathrm{mL}$ ), followed by addition of HRP-labeled goat anti-rabbit antibodies (1/10 000; Jackson ImmunoResearch, West Grove, PA, USA). Coloring reaction was performed with ortho-phenylenediamine dihydrochloride (OPD) and $\mathrm{H}_{2} \mathrm{O}_{2}$. The reaction was stopped with $4 \mathrm{M}$ $\mathrm{H}_{2} \mathrm{SO}_{4}$. Optical density (OD) was measured at $490 \mathrm{~nm}$. A reference curve to calculate ADAMTS13 concentrations was made using a normal human plasma pool at a $4 \%(\mathrm{v} / \mathrm{v})$ start dilution (NHP, $100 \%$ or $1 \mu \mathrm{g} / \mathrm{mL}$ ADAMTS13 antigen).

\section{5 | ADAMTS13 activity}

ADAMTS13 activity was measured using the Fluorescent Resonance Energy Transfer (FRET) assay with the FRETS-VWF73 substrate (Peptides International, Louisville, KY, USA). ${ }^{21}$ Briefly, plasma (3\%, $\mathrm{v} / \mathrm{v}$, diluted in HBS containing bovine serum albumin (BSA, $1 \mathrm{mg}$ / $\mathrm{mL}$ )) was added to $2 \mu \mathrm{mol} / \mathrm{L}$ FRETS-VWF73 substrate (final volume of $200 \mu \mathrm{L}) .{ }^{13}$ Proteolysis of FRETS-VWF73 by plasma ADAMTS13 was followed by measuring the change in fluorescence in function of time using a FLUOstar OPTIMA reader (BMG Labtech $\mathrm{GmbH}$, Offenburg, Germany). Thirty cycles of 2.5 minutes were measured using excitation at $355 \mathrm{~nm}$ and emission at $460 \mathrm{~nm}$. Fluorescent intensities were graphed in function of time and slopes of the linear part of the resulting curves were determined. A reference curve derived from the slopes of the plotted curves of different dilutions of NHP was used to calculate ADAMTS13 activity levels (NHP, 100\%

TAB LE 1 Clinical parameters and ADAMTS13 and von Willebrand factor levels during disease episodes and after start of prophylactic fresh frozen plasma (FFP) infusion

\begin{tabular}{|c|c|c|c|c|c|}
\hline \multicolumn{6}{|c|}{ Disease episodes } \\
\hline 1 & 1 & 34 & 1768 & & Escherichia coli, Yersinia enterocolitica \\
\hline 2 & 2 & n.d. & n.d. & & Varicella \\
\hline 6 & 3 & 14 & 6234 & Renal features & Flu-like syndrome \\
\hline 11 & 5 & 38 & 2459 & & Viral gastroenteritis \\
\hline 15 & 6 & 22 & 2670 & & Viral infection, faryngitis \\
\hline 20 & 7 & 9 & 4223 & Renal features & Viral infection \\
\hline 21 & 8 & 14 & 2611 & & Viral infection, faryngitis \\
\hline \multirow[t]{2}{*}{28} & 12 & 63 & 695 & ischemic CVA & \\
\hline & 13 & 83 & 819 & & \\
\hline \multicolumn{6}{|c|}{ Start prophylactic FFP infusion } \\
\hline \multicolumn{2}{|l|}{ Session } & \multicolumn{2}{|l|}{ FFP infusion } & Platelet count $\left(\times 10^{9} / \mathrm{L}\right)$ & LDH level (U/L) \\
\hline \multirow[t]{2}{*}{1} & & Pre & \multicolumn{2}{|r|}{179} & 636 \\
\hline & & $24 \mathrm{~h}$ post & & 206 & 553 \\
\hline \multirow[t]{2}{*}{4} & & \multicolumn{3}{|l|}{ Pre } & \\
\hline & & \multicolumn{2}{|l|}{ Post } & & \\
\hline
\end{tabular}

Platelet counts and LDH levels were determined during disease episodes and after start of prophylactic FFP infusion; "pre" indicates samples taken before FFP infusion and "post" indicates samples taken immediately after completion of the FFP infusion session (except session 1 , sample taken $24 \mathrm{~h}$ after completion). All disease episodes are clinical relapse events (severe thrombocytopenia and increased LDH levels) except for episode 10 which is a biological relapse (indicated by ${ }^{*}$ ). Associated events and known infection at time of TTP episodes are indicated. n.d.: not detectable, CVA: cerebrovascular accident. 
ADAMTS13 activity). As a negative control, $10 \mathrm{mmol} / \mathrm{L}$ EDTA was added to NHP.

\section{6 | Anti-ADAMTS13 autoantibodies}

The presence of anti-ADAMTS13 autoantibodies was detected using an immunoassay as described ${ }^{22}$, with minor modifications. A 96well microtiter plate was coated with rhADAMTS13 $(2.7 \mu \mathrm{g} / \mathrm{mL}$ in PBS). Patient plasma was added (start dilution 10\%, v/v), and a 1 in 2 dilution series was made. Bound anti-ADAMTS13 autoantibodies were detected by adding a mixture of HRP-labeled monoclonal anti$\operatorname{lgG}_{1-4}$ antibodies (anti-lgG - -HRP 1/20 000, anti-lgG $2-4$-HRP 1/2000; Sanquin Reagents, Amsterdam, The Netherlands). Coloring reaction was performed as described above. Plasma of an iTTP patient with detectable anti-ADAMTS13 autoantibodies was used as a positive control (start dilution 1\%, v/v). Data were expressed as relative OD after setting the OD value of the $1 \%$ dilution of the positive control as 1 .

\section{7 | VWF antigen}

Plasma VWF antigen levels were measured using an immunoassay as previously described. ${ }^{23}$ Briefly, a 96-well microtiter plate was coated with polyclonal rabbit anti-human VWF antibodies (1/1000 in carbonate/bicarbonate coating buffer; Dako, Glostrup, Denmark). Plasma (start dilution $1.25 \%, \mathrm{v} / \mathrm{v}$ ) was added, and a 1 in 2 dilution series was made. After washing, detection was performed using HRP-labeled polyclonal rabbit anti-human VWF antibodies (1/3000; Dako). Coloring reaction was performed as described above. A reference curve to calculate VWF antigen levels was made using a dilution series of NHP starting at a 1.25\% (v/v) dilution (NHP, 100\%, $10 \mu \mathrm{g} / \mathrm{mL}$ VWF antigen).

\section{8 | VWF multimer analysis}

Von Willebrand factor multimeric patterns were analyzed using gel electrophoresis as described. ${ }^{24,25}$ Briefly, VWF multimers present in plasma were separated on a SDS 1.2\% agarose gel. After electrophoresis, the gel was dried on a Gelbond film (Lonza, Basel, Switzerland). VWF multimer patterns were visualized using alkaline phosphatase(AP) labeled anti-VWF antibodies and AP conjugate substrate kit (Bio-Rad, Hercules, CA, USA).

\subsection{ADAMTS13 gene analysis}

Human genomic DNA was extracted from peripheral blood leukocytes from the patient and parents using standard methods. All 29 ADAMTS13 exons and intron-exon boundaries were amplified from genomic DNA via polymerase chain reaction with primers described elsewhere. ${ }^{14,26}$ After purification, all fragments were sequenced using the BigDye Terminator Cycle sequencing kit (Life Sciences, Guilford, CT, USA) on an ABI 3130XL genetic analyzer (Life Sciences).
Mutations are described according to the Human Genome Variation Society guidelines. ${ }^{27}$

\subsection{0 | Expression of wild-type and mutant rhADAMTS13 proteins}

Construction of the pc.DNA 6.1/V5-His c.1492C >T and pc.DNA 6.1/ V5-His c.768_774dup ADAMTS13 expression plasmids and subsequent transient transfection to, respectively, express recombinant proteins p.R498C ADAMTS13 and p.G259PfsX133 is described in Appendix S1.

\subsection{1 | Intracellular expression of WT and mutant rhADAMTS13}

Transient transfected Chinese Hamster Ovary (CHO K1) cells were detached 48 hours after transfection and seeded on a precoated poly-L-lysine (R\&D Systems, Oxon, UK) LabTek at 60000 cells/well. After 6 hours, when the cells were attached, cells were fixed using a $4 \%$ paraformaldehyde solution. Permeabilization was performed using Triton X-100 (0.1\% in PBS; Sigma) for 15 minutes at room temperature. After washing the cells in PBS with $0.1 \%$ Tween-20, cells were blocked with PBS containing 5\% normal goat serum (Abcam, Cambridge, UK), 1\% BSA, and 0.1\% Tween-20. WT and mutant ADAMTS13 were visualized using our in-house developed polyclonal rabbit anti-rhADAMTS13 antibody $(1 \mu \mathrm{g} /$ $\mathrm{mL}$ in PBS containing 5\% normal goat serum, 1\% BSA, and $0.1 \%$ Tween-20) and Alexa Fluor 555 labeled goat anti-rabbit antibodies $(0.5 \mu \mathrm{g} / \mathrm{mL}$ in PBS containing $5 \%$ normal goat serum, $1 \% \mathrm{BSA}$, and $0.1 \%$ Tween-20; Abcam). As a negative control for the staining, the primary antibody was omitted. Cell nuclei were visualized using 4'6-di-amidino-2-phenylindole di-lactate (DAPI; ThermoFisher Scientific). Intracellular WT or mutant ADAMTS13 and cell nuclei were visualized using a Zeiss Axio-observer Z1 epifluorescence microscope (Zeiss Microscopy, Jena, Germany) connected to a Zeiss Axiocam 506 monochromatic CCD camera (Zeiss) and pictures were processed using Zeiss ZEN 2.3 Blue software (Zeiss).

\subsection{2 | Molecular modeling of WT and mutant hADAMTS13}

Molecular models of WT and mutant hADAMTS13 were built using homology models of known ADAMTS13 domain structures ${ }^{28,29}$ and the YASARA\&Whatflf package. The p.R498C mutation was introduced with the mutagenesis module of PyMOL 1.8.2.1 (The PyMOL Molecular Graphics System, Version 1.8.2.1 Schrödinger, LLC). Molecular dynamics (MD) simulations ${ }^{30}$ allowed to perform structural comparison of the cysteine-rich and spacer domains of WT and p.R498C ADAMTS13. Changes in root-mean-square deviation (RMSD) values of heavy atoms in WT and p.R498C ADAMTS13 during a 100-ns MD simulation were calculated ${ }^{31}$, see Appendix S1. 


\subsection{3 | Statistical analysis}

Data are expressed as mean \pm standard deviation (SD). Statistical analysis was performed using one-way ANOVA with Dunnett's multiple comparison test. Values of $P<.05$ were considered statistically significant. All statistical analysis was performed using GraphPad Prism v5.03 software (GraphPad Software, Inc., San Diego, CA, USA).

\section{3 | RESULTS}

\subsection{Congenital TTP diagnosis and clinical evolution}

At the age of 20, the patient relapsed again (episode 7, Table 1). As his clinical history was indicative for TTP, ADAMTS13 characteristics were tested. Indeed, ADAMTS13 activity and antigen levels were undetectable. Both, mother and father were asymptomatic with ADAMTS13 activities of, respectively, 37.8\% (mean of $n=3$ ) and $18.3 \%$ (mean of $n=2$ ) and ADAMTS13 antigen levels of, respectively, $48.7 \%$ and $49.7 \%$ (mean of $n=3$ ). The patient did not have any detectable anti-ADAMTS13 autoantibodies in his plasma. The absence of ADAMTS13 activity, antigen, and anti-ADAMTS13 autoantibodies together with the fact that the patient was symptomatic since childhood led to the diagnosis of congenital TTP. This was confirmed by sequence analysis of the ADAMTS13 gene with identification of 2 compound heterozygous mutations: the c.768_774dup frameshift mutation (p.G259PfsX133) ${ }^{32}$ and the c.1492C >T ADAMTS13 missense mutation (p.R498C) ${ }^{10}$ (Figure 1A). The 7 nucleotides (CCGCGCC) duplicated in the metalloprotease domain (amino acid, AA 80-286) ${ }^{28}$ induce a frameshift resulting in a premature stop codon in the first thrombospondin type 1 repeat of ADAMTS13 (p. G259PfsX133) (Figure 1A). The point mutation is located in the cysteine-rich domain and results in the p.R498C amino acid substitution (Figure $1 \mathrm{~A}$ ). The mother and father of the patient are both heterozygous carriers of the p.G259PfsX133 and the p.R498C mutation, respectively.

Since the age of 20, the patient relapsed 6 times (episode 8-13) of which 5 were clinical relapses (episodes $8,9,11-13)^{33}$ associated with severe thrombocytopenia and increased LDH levels (Table 1). As expected, ADAMTS13 activity and antigen levels were severely reduced (episode 8 , Figure 1B,C). At the age of 25 (episode 10 , Table 1), the patient presented with an ischemic cerebrovascular accident (CVA) which was accompanied by severely reduced ADAMTS13 activity and antigen levels (Figure 1B,C) but no presence of clinical signs and/or symptoms of TTP as platelet counts and LDH levels were not below or above the reference ranges. As expected, UL-VWF multimers disappeared during these relapse episodes and VWF antigen levels remained normal (episode 8 and 10, Figure 1D,E). At the age of 28 (episode 12, Table 1), the patient experienced a second CVA associated with a clinical TTP relapse (severe thrombocytopenia and increased LDH levels).

Due to the occurrence of multiple CVA's, it was decided to treat the patient prophylactically with infusion of 3 units of FFP approximately every 3 weeks. Platelet counts increased (Table 1) and ADAMTS13 activity and antigen levels slightly increased after each FFP infusion (Figure 1B,C). VWF antigen levels remained constant (Figure 1E), and UL-VWF multimers were often decreased after FFP infusion (Figure 1D). Apparently, even these small ADAMTS13 increases can sufficiently reduce pathologic UL-VWF multimers. Prophylactic treatment has been successful as the patient remains asymptomatic without recurrent minor or major neurological incidents.

\section{2 | The p.R498C and p.G259PfsX133 ADAMTS13 mutations cause severe ADAMTS13 secretion and translation defects in vitro, respectively}

To investigate the effect of the p.R498C and the p.G259PfsX133 mutations on ADAMTS13 secretion, we introduced each mutation separately in a WT ADAMTS13 expression vector and performed transient transfections in $\mathrm{CHO} \mathrm{K} 1$ cells. In addition, we co-transfected the cells with both p.R498C and p.G259PfsX133 mutants to mimic the compound heterozygous nature of the patient. Co-transfection with a GFP-expressing plasmid in all experiments allowed evaluation of the transfection efficiency. Transfection efficiencies for all mutants were not significantly different from WT (data not shown). The expression levels of p.R498C ADAMTS13 and p.R498C + p.G259PfsX133 ADAMTS13 were severely reduced $(39.1 \pm 19.6 \%, P<.01$ and $29.2 \pm 21.7 \%, P<.001$, respectively; Figure $2 \mathrm{~A}$ ) compared to the expression levels of WT ADAMTS13 $(100 \pm 15.1 \%$; Figure $2 A)$. In contrast, the p.G259PfsX133 mutant was not detected in the expression medium (Figure $2 A$ ). In line with this, rhADAMTS13 protein could be visualized via fluorescence microscopy in permeabilized transfected $\mathrm{CHO}$ K1 cells with the p.R498C, the p.R498C + p.G259PfsX133, and WT ADAMTS13 while the p.G259PfsX133 mutant could not (Figure 2B). These data indicate that the p.R498C mutation impairs secretion and that the p.G259PfsX133 mutation, which results in a frameshift mutation at the end of the metalloprotease domain (AA 259 of $80-286^{28}$ ) of ADAMTS13, probably leads to an unstable RNA molecule that impedes translation of the protein.

\section{3 | The p.R498C mutation destabilizes the cysteine-rich domain}

Molecular modeling of WT and mutant p.R498C MDTCS revealed that the p.R498C mutation results in loss of hydrogen bonds donated by the R498 residue (Figure 3). The R498 side chain makes extensive contacts with neighboring residues (hydrogen bonds with T453, Q456, and H485 and a cation-pi interaction with W470; Figure 3A, middle panel) which collectively contribute to the stability of the cysteine-rich domain. These hydrogen bonds are present throughout the 100 ns MD simulations for the WT MDTCS model. The calculated RMSD values over the MD simulation trajectories of both WT and the R498C mutant allowed a comparative analysis on the conformation of the WT and p.R498C MDTCS (Figure 3A,B, right 
(A)

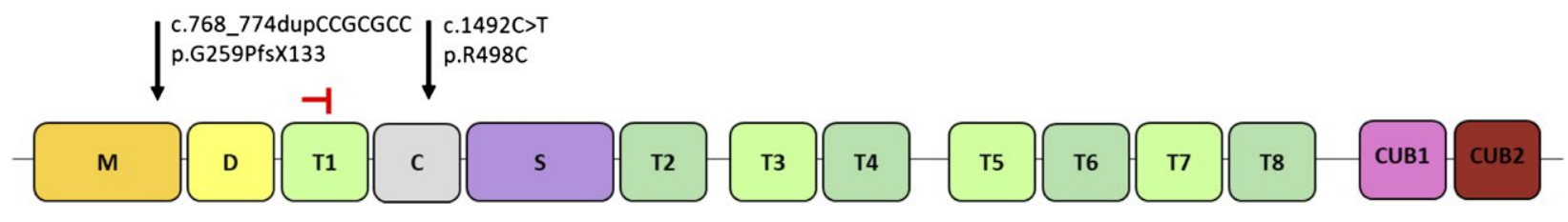

(B)

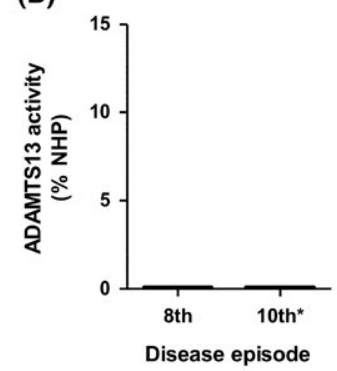

(D)

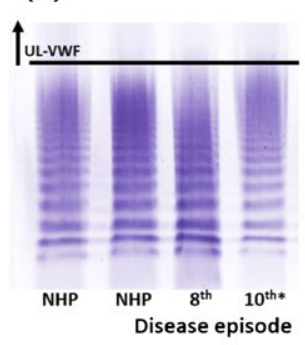

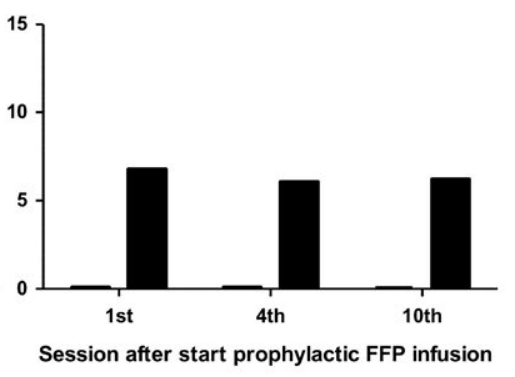

Session after start prophylactic FFP infusion

(C)
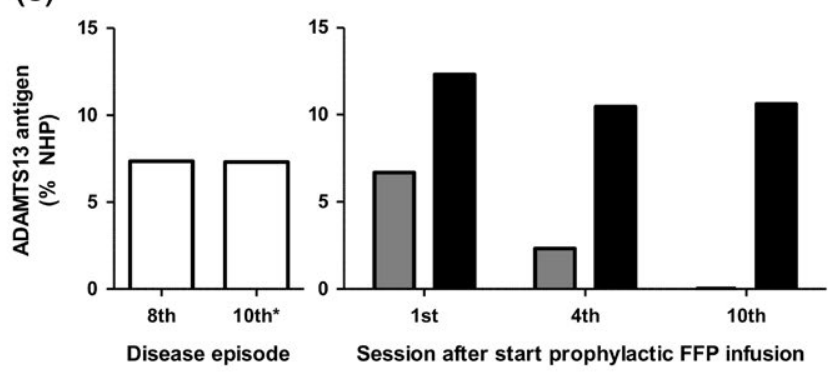

(E)
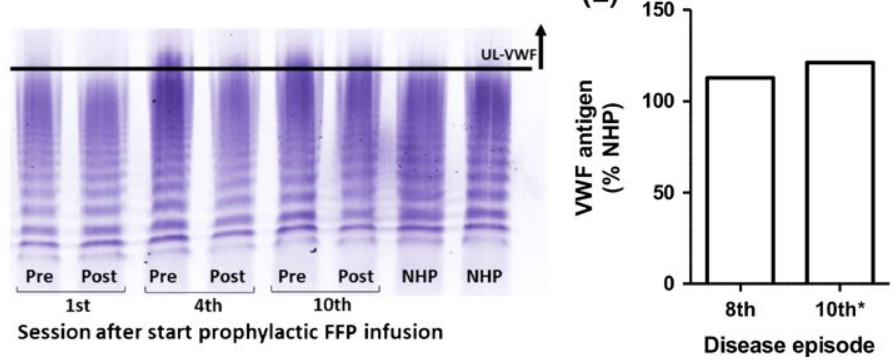

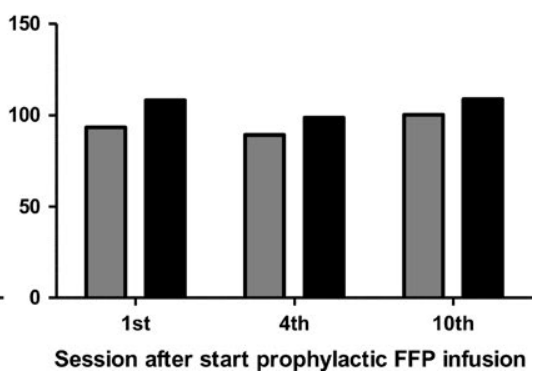

FIGURE 1 ADAMTS13 and von Willebrand factor (VWF) parameters during TTP diagnosis and disease course. A, Localization of the p.G259PfsX133 and p.R498C mutations in the ADAMTS13 gene. ADAMTS13 domain structure with the metalloprotease domain (M, AA 80-286), disintegrin-like domain (D, AA 287-383), first thrombospondin type 1 repeat (T1, AA 384-439), cysteine-rich domain (C, AA 440556), spacer domain (S, AA 556-685), 7 additional T domains (T2-T8, AA 682-1131), and 2 CUB domains (AA 1192-1427). The c.768_774dup frameshift mutation (p.G259PfsX133) is located in the $M$ domain and induces a frameshift resulting in a premature stop codon in T1. The c.1492C $>T$ ( p.R498C) missense mutation is located in the cysteine-rich domain. B-E, ADAMTS13 activity, ADAMTS13 antigen, UL-VWF multimers, and VWF antigen during disease episodes and after start of prophylactic fresh frozen plasma (FFP) infusion. Plasma samples during clinical relapse episode (8th, episode 8, Table 1) and biological relapse episode (10th*, episode 10*, Table 1) (white bars) and after start of prophylactic FFP infusion; "Pre" (gray bars) indicates samples taken before FFP infusion and "Post" (black bars) indicates samples taken immediately after completion of the FFP infusion session (except session 1, sample taken $24 \mathrm{~h}$ after completion), were analyzed for ADAMTS13 activity levels using the FRETS assay (B), ADAMTS13 antigen levels using an ADAMTS13 antigen ELISA (C), for the presence of UL-VWF multimers using VWF multimer analysis (D), and VWF antigen levels using the VWF antigen ELISA (E). Data are represented as \% $\mathrm{NHP}$ (mean, $\mathrm{n}=3$ )

panel). The effects of the altered intra-domain interactions of the cysteine-rich domain became visible after $\sim 54 \mathrm{~ns}$ of simulation time for the R498C according to highest peak in RMSD values (Figure 3B, right panel). The extracted snapshots from this specific time revealed that R498C mutant is not as structurally stable as WT MDTCS. Only the hydrogen bond between R498C and H485 was observed, showing that the cysteine-rich domain structure is significantly distorted in p.R498C MDTCS as compared to WT (Figure 3). The absence of these interactions in p.R498C MDTCS structure leads to the formation of a less stable p.R498C cysteine-rich domain.

\section{4 | DISCUSSION}

We here report a case study of a male patient suffering from childonset congenital TTP but only diagnosed during adulthood. The patient was found to be compound heterozygous for the p.R498C and the p.G259PfsX133 ADAMTS13 mutations. The ADAMTS13 mutations severely impair ADAMTS13 secretion and translation in vitro, respectively, explaining the patient's phenotype. Due to exacerbations of relapses and occurrence of ischemic cerebrovascular accidents, the patient is now treated prophylactically with FFP and is free of new TTP episodes.

TTP diagnosis is very challenging as the clinical spectrum can be very distinct (microangiopathic hemolytic anemia and thrombocytopenia with/without renal, abdominal, cardiac, and/or neurological features ranging from confusion till coma) and overlaps with other thrombotic microangiopathies (TMAs). ${ }^{1,2}$ However, severe ADAMTS13 deficiency is specific for TTP and is suited to distinguish TTP from other TMA disorders. ${ }^{34}$ Also in our study, ADAMTS13 testing led to a correct diagnosis of TTP. Moreover, severe ADAMTS13 deficiency requires plasma infusion in contrast to other TMAs. ${ }^{35}$ 
(A)

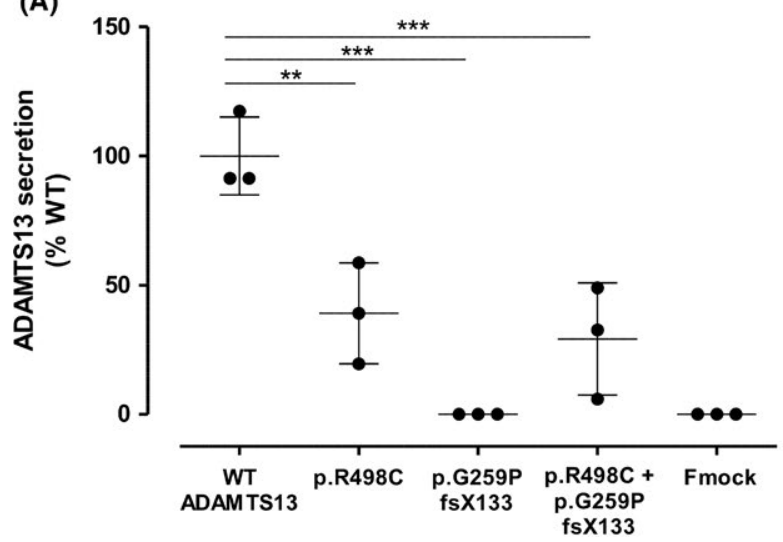

(B)
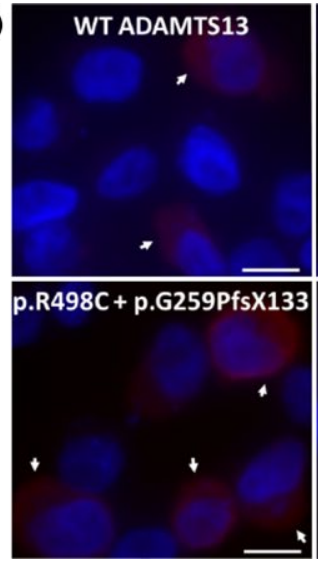

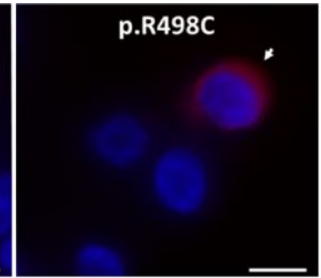

Fmock

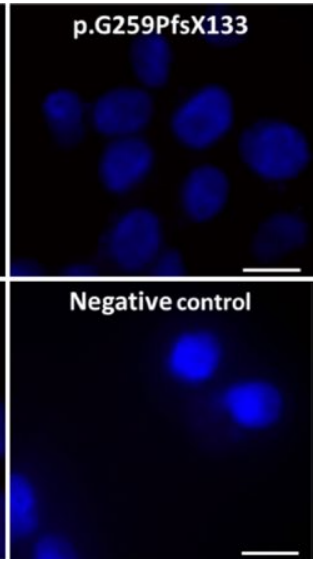

FIGURE 2 Secretion and translation defects of the p.R498C and p.G259PfsX133 mutants, respectively. A, Expression levels of ADAMTS13 WT and mutants in medium of transiently transfected CHO K1 cells were determined via ADAMTS13 antigen ELISA. Expression medium of Fmock cells was used as a negative control. Data are represented in \% WT ADAMTS13 secretion (mean \pm SD, $n=3$ with ${ }^{* *} P<.01$ and $\left.{ }^{* * *} P<.001\right)$. B, Fluorescent staining of intracellular WT and mutant ADAMTS13 using the polyclonal anti-rhADAMTS13 antibody and an ALEXA Fluor 555 labeled goat anti-rabbit IgG (red, white arrow). Cell nuclei were stained with DAPI (blue). As a negative control, the primary antibody was omitted. Pictures were taken using a Zeiss Axio-observer Z1 epifluorescence microscope. Scale bar represents $10 \mu \mathrm{m}$

FIGURE 3 Structural comparison of WT and p.R498C MDTCS. Molecular modeling of the $\mathrm{p} . \mathrm{R} 498 \mathrm{C}$ mutation shows structural deviation in the ADAMTS13 cysteine-rich domain. 3D structure of WT (A, left panel) and p.R498C (B, left panel) ADAMTS13 cysteine-rich and spacer domain at 54ns MD, R498 (A, middle panel), and p.R498C ( $B$, middle panel) intra-domain interactions in detail and changes in RMSD values of heavy atoms of WT (A, right panel) and p.R498C (B, right panel) ADAMTS13 cysteine-rich domain during a 100ns MD simulation
(A)

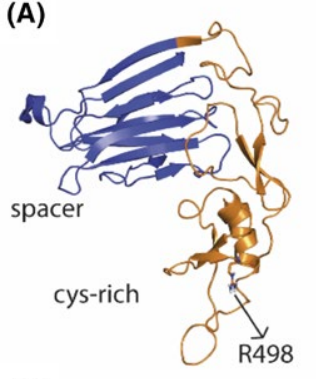

(B)

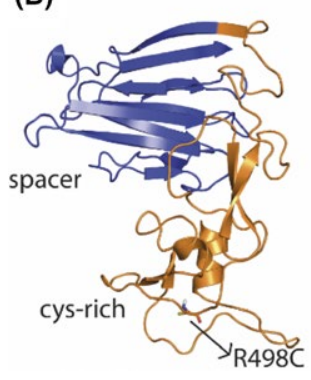

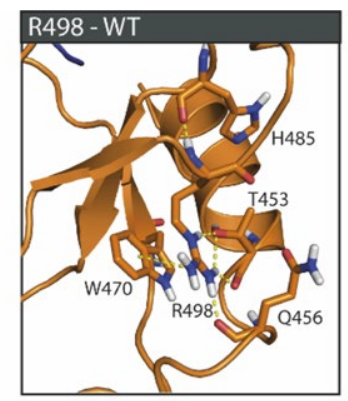

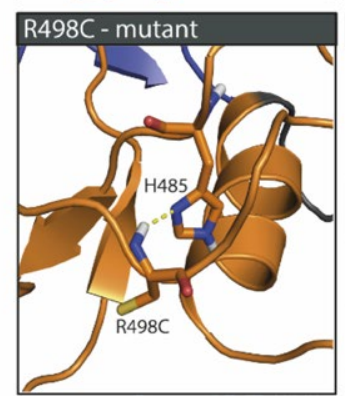

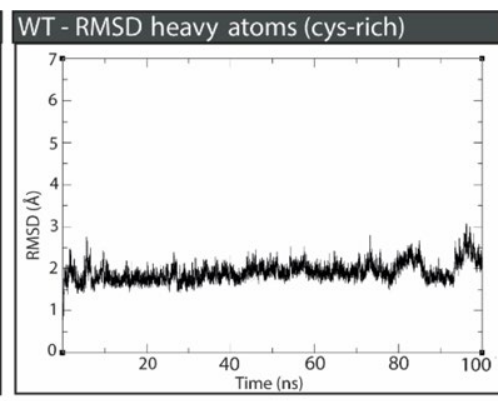

R498C - RMSD heavy atoms (cys-rich)

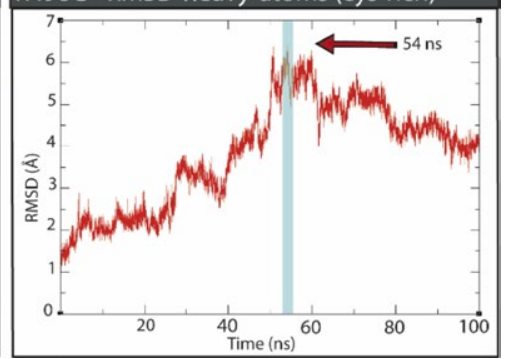

Without prompt plasma infusion treatment, TTP mortality is still 10$20 \% .{ }^{1}$ Identification of TTP and appropriate treatment is crucial to reverse signs and symptoms and to prevent permanent renal and/ or neurological injury and additionally, patients can be monitored to prevent future relapse. $^{2}$

To date, the p.R498C ADAMTS13 point mutation has been reported in a compound heterozygous woman with pregnancyassociated TTP together with the p.R1060W mutation; however, the functional effect was only characterized in vitro for the p.R1060W but not for the p.R498C mutation. ${ }^{10,16}$ Our in vitro data show that the single amino acid change results in a significant reduction in p.R498C ADAMTS13 secretion compared to WT ADAMTS13. The secretion defect caused by the p.R498C mutation is also reflected in the father's phenotype, who is carrier of the p.R498C mutation, as his plasma ADAMTS13 antigen levels are 49.7\%. The R498 residue is located in the cysteine-rich domain of ADAMTS13. The side chain of the R498 is buried and important for intra-domain interactions, assuring the protein's stability. ${ }^{29}$ The $\mathrm{R} 498 \mathrm{C}$ conversion predicts protein misfolding and may activate an unfolded protein response in the endoplasmic reticulum, resulting in degradation of the misfolded protein after its export from the endoplasmic reticulum to the cytosol. ${ }^{36}$

The p.G259PfsX133 frameshift mutation has been reported previously in a compound heterozygous woman with pregnancyassociated TTP together with the p.R1060W mutation. ${ }^{32}$ Only a few ADAMTS13 duplications have been described ${ }^{5,9,37-41}$ as the majority of known ADAMTS13 mutations are point mutations resulting in a single amino acid change. The duplication of 7 nucleotides 
(CCGCGCC), associated with a frameshift, induces a premature stop codon in the first thrombospondin type 1 motif, resulting in the truncated p.G259PfsX133 ADAMTS13 mutant. We investigated the effect of the frameshift mutation on ADAMTS13 levels. The p.G259PfsX133 ADAMTS13 was not detected in the expression medium of the transfected $\mathrm{CHO}$ K1 cells, nor was it intracellularly stained using the polyclonal anti-rhADAMTS13 antibodies. The RNA of the truncated ADAMTS13 mutant is most probably unstable and therefore not translated. These in vitro data are in agreement with the mother's phenotype, who is carrier of the p.G259PfsX133 mutation and has 48.7\% ADAMTS13 antigen levels in her plasma.

In the patient's history, we note 12 episodes of acute TTP up till now. Interestingly, these episodes were always triggered by either a viral or bacterial infection or stress which have been abundantly suggested as potential provoking factors for TTP. ${ }^{2}$ Remarkably, during disease course, the patient experienced 2 ischemic cerebrovascular accidents of which the last was associated with TTP relapse. It is known that a wide spectrum of neurological manifestations can occur during TTP, from seizures to coma, although transient symptoms ranging from headache to focal signs are more common. ${ }^{1}$ It has been suggested that characteristic neurovascular complications of TTP may develop before the onset of alarming hematologic abnormalities (severely reduced platelet counts, hemolytic anemia, increased LDH levels). ${ }^{42}$ Local prothrombotic changes together with chronic low ADAMTS13 levels may be significant enough to cause focal platelet aggregation without permanently overcoming the systemic antithrombotic mechanisms. This could explain the lag between stroke presentation and the development of the typical hematologic features of TTP seen in all patients. As the patient's TTP relapse rate increased and cerebrovascular accidents occurred, it was decided to start prophylactic FFP infusions. As reported, initiation of (lifelong) prophylactic FFP infusion prevents relapses and decreases mortality. ${ }^{43}$ Since the start of prophylactic FFP, the patient remained clinically in remission.

In conclusion, we report a congenital male TTP patient being compound heterozygous for the $\mathrm{p} . \mathrm{R} 498 \mathrm{C}$ point mutation and the p.G259PfsX133 frameshift mutation with no detectable ADAMTS13 activity in the plasma. Indeed, we showed that p.R498C severely impairs secretion and p.G259PfsX133 impairs translation of ADAMTS13 explaining the patient's phenotype.

\section{AUTHORS CONTRIBUTIONS}

A.S. Schelpe designed and performed experiments, analyzed and interpreted the data, and wrote the manuscript; C. Geeroms, I. Pareyn, N. Vandeputte, L.C. Velásquez Pereira, and E. Roose performed experiments; Dr. Karel Forstier gave clinical input, Dr. K. Jochmans, and $\mathrm{C}$. Orlando provided the patient plasma samples, wrote the case history section, and critically reviewed the manuscript; $\mathrm{H}$. Deckmyn and S.F. De Meyer critically reviewed the manuscript; $\mathrm{K}$. Vanhoorelbeke designed the experiments, interpreted the data, wrote the manuscript, and provided funding.

\section{CONFLICT OF INTEREST}

All other authors declare no competing financial interests.

\section{ORCID}

An-Sofie Schelpe iD http://orcid.org/0000-0002-3059-364X

\section{REFERENCES}

1. Kremer Hovinga JA, Coppo P, Lämmle B, Moake J, Miyata T, Vanhoorelbeke K. Thrombotic thrombocytopenic purpura. Nat Rev Dis Primers. 2017;3:1-17.

2. Joly BS, Coppo P, Veyradier A. Thrombotic thrombocytopenic purpura. Blood. 2017;129:2836-2846.

3. Joly BS, Vanhoorelbeke K, Veyradier A. Understanding therapeutic targets in thrombotic thrombocytopenic purpura. Intensive Care Med. 2017;43:1398-1400.

4. Scully M, Hunt BJ, Benjamin S, et al. Guidelines on the diagnosis and management of thrombotic thrombocytopenic purpura and other thrombotic microangiopathies. $\mathrm{Br} J$ Haematol. 2012;158:323-335.

5. Levy GG, Nichols WC, Lian EC, et al. Mutations in a member of the ADAMTS gene family cause thrombotic thrombocytopenic purpura. Nature. 2001;413:488-494.

6. Kokame K, Matsumoto M, Soejima K, et al. Mutations and common polymorphisms in ADAMTS13 gene responsible for von Willebrand factor-cleaving protease activity. Proc Natl Acad Sci U S A. 2002;99:11902-11907.

7. Fujimura $\mathrm{Y}$, Matsumoto $M$, Isonishi $A$, et al. Natural history of Upshaw-Schulman syndrome based on ADAMTS13 gene analysis in Japan. J Thromb Haemost. 2011;9(Suppl 1):283-301.

8. Veyradier A, Lavergne J-M, Ribba A-S, et al. Ten candidate ADAMTS13 mutations in six French families with congenital thrombotic thrombocytopenic purpura (Upshaw-Schulman syndrome). J Thromb Haemost. 2004;2:424-429.

9. von Krogh AS, Quist-Paulsen P, Waage A, et al. High prevalence of hereditary thrombotic thrombocytopenic purpura in central Norway: from clinical observation to evidence. J Thromb Haemost. 2016;14:73-82.

10. Scully $M$, Thomas M, Underwood M, et al. Thrombotic thrombocytopenic purpura and pregnancy: presentation, management, and subsequent pregnancy outcomes. Blood. 2014;124:211-219.

11. Plaimauer B, Fuhrmann J, Mohr G, et al. Modulation of ADAMTS13 secretion and specific activity by a combination of common amino acid polymorphisms and a missense mutation. Blood. 2006;107:118-125.

12. Underwood M, Peyvandi F, Garagiola I, Machin S, Mackie I. Degradation of two novel congenital TTP ADAMTS13 mutants by the cell proteasome prevents ADAMTS13 secretion. Thromb Res. 2016;147:16-23.

13. De Cock E, Hermans C, De Raeymaecker J, et al. The novel ADAMTS13-p.D187H mutation impairs ADAMTS13 activity and secretion and contributes to thrombotic thrombocytopenic purpura in mice. J Thromb Haemost. 2015;13:283-292.

14. Feys HB, Pareyn I, Vancraenenbroeck R, et al. Mutation of the $\mathrm{H}$-bond acceptor S119 in the ADAMTS13 metalloprotease domain reduces secretion and substrate turnover in a patient with congenital thrombotic thrombocytopenic purpura. Blood. 2009;114:4749-4752.

15. Peyvandi F, Lavoretano S, Palla R, et al. Mechanisms of the interaction between two ADAMTS13 gene mutations leading to severe deficiency of enzymatic activity. Hum Mutat. 2006;27:330-336. 
16. Camilleri RS, Cohen H, Mackie IJ, et al. Prevalence of the ADAMTS-13 missense mutation R1060W in late onset adult thrombotic thrombocytopenic purpura. J Thromb Haemost. 2008;6:331-338.

17. Roose E, Schelpe AS, Joly BS, et al. An open conformation of ADAMTS-13 is a hallmark of acute acquired thrombotic thrombocytopenic purpura. J Thromb Haemost. 2018;16:378-388.

18. Chauhan AK, Motto DG, Lamb CB, et al. Systemic antithrombotic effects of ADAMTS13. J Exp Med. 2006;203:767-776.

19. Feys HB, Liu F, Dong N, et al. ADAMTS-13 plasma level determination uncovers antigen absence in acquired thrombotic thrombocytopenic purpura and ethnic differences. J Thromb Haemost. 2006;4:955-962.

20. Feys HB, Roodt J, Vandeputte N, et al. Thrombotic thrombocytopenic purpura directly linked with ADAMTS13 inhibition in the baboon (Papio ursinus). Blood. 2010;116:2005-2010.

21. Kokame K, Nobe $\mathrm{Y}$, Kokubo $\mathrm{Y}$, Okayama A, Miyata T. FRETSVWF73, a first fluorogenic substrate for ADAMTS13 assay. $\mathrm{Br} J$ Haematol. 2005;129:93-100.

22. Pos W, Sorvillo N, Fijnheer R, et al. Residues arg568 and phe592 contribute to an antigenic surface for anti-adamts13 antibodies in the spacer domain. Haematologica. 2011;96:1670-1677.

23. Vanhoorelbeke K, Cauwenberghs N, Vauterin S, Schlammadinger A, Mazurier C, Deckmyn H. A reliable and reproducible ELISA method to measure ristocetin cofactor activity of von Willebrand Factor. Thromb Haemost. 2000;83:107-113.

24. Ruggeri ZM, Zimmerman TS. The complex multimeric composition of factor VIII/von Willebrand Factor. Blood. 1981;57:1140-1143.

25. De Meyer SF, Vandeputte N, Pareyn I, et al. Restoration of plasma von Willebrand factor deficiency is sufficient to correct thrombus formation after gene therapy for severe von Willebrand disease. Arterioscler Thromb Vasc Biol. 2008;28:1621-1626.

26. Kokame K, Miyata T, Moschcowitz E, et al. Genetic defects leading to hereditary thrombotic thrombocytopenic purpura. Semin Hematol. 2004;41:34-40.

27. den Dunnen JT, Dalgleish R, Maglott DR, et al. HGVS recommendations for the description of sequence variants: 2016 update. Hum Mutat. 2016;37:564-569.

28. Ercig B, Wichapong K, Reutelingsperger C, Vanhoorelbeke K, Voorberg J, Nicolaes G. Insights into 3D structure of ADAMTS13: a stepping stone towards novel therapeutic treatment of thrombotic thrombocytopenic purpura. Thromb Haemost. 2018;118:028-041.

29. Akiyama M, Takeda S, Kokame K, Takagi J, Miyata T. Crystal structures of the noncatalytic domains of ADAMTS13 reveal multiple discontinuous exosites for von Willebrand factor. Proc Natl Acad Sci U S A. 2009;106:19274-19279.

30. Case DA, Ben-Shalom IY, Brozell SR, et al. York and P.A. Kollman (2018), AMBER 2018, University of California, San Francisco.

31. Roe DR, Cheatham III TE. PTRAJ and CPPTRAJ: software for processing and analysis of molecular dynamics trajectory data. J Chem Theory Comput. 2013;9:3084-3095.

32. Kentouche K, Voigt A, Schleussner E, et al. Pregnancy in UpshawSchulman syndrome. Hamostaseologie. 2013;33:144-148.
33. Scully M, Cataland S, Coppo P, et al. Consensus on the standardization of terminology in thrombotic thrombocytopenic purpura and related thrombotic microangiopathies. J Thromb Haemost. 2017;15:312-322.

34. Sadler JE. What's new in the diagnosis and pathophysiology of thrombotic thrombocytopenic purpura. Hematology Am Soc Hematol Educ Program. 2015;2015:631-636.

35. George JN, Nester CM. Syndromes of thrombotic microangiopathy. N Engl J Med. 2014;371:654-666.

36. Ellgaard L, Helenius A. Quality control in the endoplasmic reticulum. Nat Rev Mol Cell Biol. 2003;4:181-191.

37. Conboy E, Partain PI, Warad D, et al. A severe case of congenital thrombotic thrombocytopenia purpura resulting from compound heterozygosity involving a novel ADAMTS13 pathogenic variant. J Pediatr Hematol Oncol. 2018;40:60-62.

38. Donadelli R, Banterla F, Galbusera M, et al. In-vitro and in-vivo consequences of mutations in the von Willebrand factor cleaving protease ADAMTS13 in thrombotic thrombocytopenic purpura. Thromb Haemost. 2006;96:454-464.

39. Krabbe JG, Kemna EWM, Strunk ALM, et al. Adult-onset congenital thrombotic thrombocytopenic purpura caused by a novel compound heterozygous mutation of the ADAMTS13 gene. Int J Hematol. 2015;102:477-481.

40. Hrachovinova I, Rittich S, Salaj P, et al. Hereditary form of thrombotic thrombocytopenic purpura. Cas Lek Cesk. 2006;145:390-392.

41. Kentouche K, Budde U, Furlan M, Scharfe V, Schneppenheim R, Zintl F. Remission of thrombotic thrombocytopenic purpura in a patient with compound heterozygous deficiency of von Willebrand factor-cleaving protease by infusion of solvent/detergent plasma. Acta Paediatr. 2002;91:1056-1059.

42. Rojas JC, Banerjee C, Siddiqui F, Nourbakhsh B, Powell CM. Pearls and oy-sters: acute ischemic stroke caused by atypical thrombotic thrombocytopenic purpura. Neurology. 2013;80:e235-e238.

43. Loirat C, Coppo P, Veyradier A. Thrombotic thrombocytopenic purpura in children. Curr Opin Pediatr. 2013;25:216-224.

\section{SUPPORTING INFORMATION}

Additional supporting information may be found online in the Supporting Information section at the end of the article.

How to cite this article: Schelpe AS, Orlando C, Ercig B, et al. Child-onset thrombotic thrombocytopenic purpura caused by p.R498C and p.G259PfsX133 mutations in ADAMTS13. Eur J Haematol. 2018;101:191-199. https://doi.org/10.1111/ ejh.13094 